\title{
Light-scattering spectra of supercooled molecular liquids
}

\author{
T. Franosch, ${ }^{1, *}$ M. Fuchs, ${ }^{1}$ and A. Latz ${ }^{2}$ \\ ${ }^{1}$ Physik-Department, Technische Universität München, D-85747 Garching, Germany \\ ${ }^{2}$ Institut für Physik, Johannes Gutenberg Universität, Staudinger Weg 7, D-55099 Mainz, Germany
}

(Received 21 June 2000; published 25 May 2001)

\begin{abstract}
The light-scattering spectra of molecular liquids are derived within a generalized hydrodynamics. The wave-vector and scattering-angle dependencies are given in the most general case and the change of the spectral features from liquid to solidlike is discussed without phenomenological model assumptions for (general) dielectric systems without long-ranged order. Exact microscopic expressions are derived for the frequency dependent transport kernels, generalized thermodynamic derivatives, and the background spectra.
\end{abstract}

PACS number(s): 64.70.Pf, 78.35.+c

\section{INTRODUCTION}

Light scattering is a powerful tool to study the dynamics of dense (transparent) materials [1]. The fluctuations of the dielectric tensor for wave-vector transfer $\mathbf{q}$ are measured, where the corresponding wavelength can be considered large compared to molecular length scales. In this case the theoretical description of the light spectra simplifies as it suffices to determine the lowest orders in wave vector $\mathbf{q}$ only. The wave vector and scattering-angle dependence of the scattering cross sections thus can be determined.

Focusing on low frequencies, the hydrodynamic approach can be used to calculate the spectral shapes. For the polarized-light-scattering spectra of gases and liquids, the assumption that dielectric fluctuations are dominantly caused by density fluctuations leads to the well-known RayleighBrillouin spectra. The corresponding hydrodynamic depolarized spectra were first obtained within simplified models by Andersen and Pecora [2] and Keyes and Kivelson [3], in the latter case, under the assumption that fluctuations in the orientations of the molecules cause the dielectric variations. As observed experimentally in molecular liquids, a negative central line appears called "Rytov dip" [4,5].

The spectra, in particular the depolarized ones, change qualitatively if the relaxation times of the structural dynamics increase upon cooling the liquids. For the polarized spectra, Mountain introduced a frequency-dependent longitudinal viscosity in order to model the additionally appearing central line [6]. In the depolarized-light-scattering spectra transverse sound peaks become visible as expected from hydrodynamic calculations for solids [7]. Within phenomenological models including nonhydrodynamic variables, the changes of the spectra from liquidlike at high to solidlike at low temperatures could be explained [8-13]. However, assumptions about the included slow variables and about phenomenological kinetic equations coupling their time dependencies, were required. The introduction of memory functions, in most detail in the recent work of Dreyfus et al. $[12,13]$, has relaxed the requirement to identify and include all slow variables but still uses phenomenological equations to couple rotational

\footnotetext{
*Present address: Lyman Laboratory of Physics, Harvard University, Cambridge, MA 02138.
}

and translational degrees of freedom.

Here, we clarify that generalized hydrodynamics and symmetry considerations suffice to explain the lightscattering spectra qualitatively as they change from liquid to solidlike. The results will be derived without any assumptions about nonhydrodynamic variables (and their couplings) and will also not depend on specific light-scattering mechanisms nor molecular parameters like shape, dipole moment, polarizabilities nor chirality. The set of slow variables we consider are the standard slow hydrodynamic variables of liquids, density $n(\mathbf{q})$, current density $\mathbf{j}(\mathbf{q})$, and temperature $\Theta(\mathbf{q})$ (connected to energy conservation), and the slow structural relaxation of supercooled liquids enters via a few memory functions. We thus provide a general framework for the analysis of light-scattering spectra in supercooled molecular liquids, which we expect will prove useful either for phenomenological discussions using fit functions for the memory kernels-we will list all restrictions on these fit functions-or for the consideration of specific scattering mechanisms. Our central technique for simplifying the spectra consists in small-wave-vector expansions of the memory functions as should be appropriate for disordered systems. Thus we adopt the idea of generalized hydrodynamics, which extends the regular hydrodynamic approach to larger frequencies. In detail, we use the one suggested by Götze and Latz [14] as it provides a physically reasonable description of glassy systems. Finally, we also derive Green-Kubo formulas, which enable e.g., computer simulations, to determine the memory functions and thus the complete spectra directly.

Our assumptions pertain to the systems under consideration and can be tested experimentally: We consider only the lowest nontrivial orders in wave-vector transfer $q$, in order to find general results for the light scattering from amorphous, dielectric, macroscopically isotropic and optically inactive materials within the framework of linear response, classical statistical mechanics, and classical electromagnetism. The condition $q a \ll 1$, where $a$ denotes either a typical molecular size, the average particle distance, or a collective correlation length, appears well satisfied for supercooled molecular liquids but excludes studies of critical phenomena. Electromagnetic retardation also can be neglected for $\omega \ll c q$, where $c$ is the speed of light.

The general formulas for the spectra and constitutive equations are presented in Sec. II. Section III lists the central 
results that are discussed in Sec. IV. The results for the depolarized spectra are compared to previous theoretical approaches in Sec. V, and conclusions in Sec. VI summarize our results. More technical aspects are contained in Appendices $\mathrm{A}$ and $\mathrm{B}$, and Appendix $\mathrm{C}$ outlines the application of our general results to specific light-scattering mechanisms.

\section{GENERAL FORMULAS}

\section{A. Dielectric fluctuations}

In a light-scattering experiment a laser beam at frequency $\omega_{i}$ induces a polarization in a transparent sample, which starts to radiate. For a homogeneous sample the radiated waves interfere constructively only in the forward direction at the same frequency as the incident wave. However, the dielectric permeability fluctuates in space and time around its average and therefore additionally a (diffusive) scattering occurs. In general, this scattering spectrum reflects the dynamical processes in the sample and depends on the frequency of the incident, $\omega_{i}$, as well as the scattered wave, $\omega_{f}$. A simplification is possible if one considers only small frequency shifts, $\omega=\omega_{i}-\omega_{f}$ with $|\omega| \ll \omega_{i}$. Then dynamical dielectric fluctuations $\left(\epsilon_{i j}(\mathbf{q}, t) \mid \epsilon_{k l}(\mathbf{q})\right)$ determine the scattering cross sections completely [7]. Here the Kubo scalar product, $(A(t) \mid B)=\left(1 / k_{B} T\right)\left\langle\delta A(t)^{*} \delta B\right\rangle$, is used, with $T$ temperature and $k_{B}$ Boltzmann's constant.

The fluctuation $\delta \epsilon_{i j}(\mathbf{q}, t)$ has even time-reversal symmetry, is a symmetric tensor of second rank, and, as Fourier transform of a real quantity, fulfills $\epsilon_{i j}(-\mathbf{q}, t)=\epsilon_{i j}(\mathbf{q}, t)^{*}$. In particular, the long-wavelength limit $\epsilon_{i j}(\mathbf{q} \longrightarrow 0, t)$ is real. Different Cartesian components $i, j, k, l$ are picked out depending on the polarization directions of the incoming and scattered light [1]; see Appendix A for more details. The dynamical evolution is given by the Liouvillian $\mathcal{L}$ via $\partial_{t} A$ $=i \mathcal{L} A$. A Laplace transformation-convention $f(z)$ $=i \int_{0}^{\infty} d t e^{i z t} f(t)$ for $\mathfrak{I} z>0$-thus leads to the problem to calculate, for $q \rightarrow 0$, the matrix elements of:

$$
\left(\boldsymbol{\epsilon}_{i j}(\mathbf{q}, z) \mid \boldsymbol{\epsilon}_{k l}(\mathbf{q})\right)=\left(\boldsymbol{\epsilon}_{i j}(\mathbf{q})\left|\frac{1}{\mathcal{L}-z}\right| \boldsymbol{\epsilon}_{k l}(\mathbf{q})\right) .
$$

The spectra at frequency $\omega$ then are given by the imaginary part of $\left(\epsilon_{i j}(\mathbf{q}, \omega+i 0) \mid \epsilon_{k l}(\mathbf{q})\right)$ denoted by $\left(\epsilon_{i j}(\mathbf{q}, \omega) \mid \epsilon_{k l}(\mathbf{q})\right)^{\prime \prime}$.

\section{B. Generalized hydrodynamics}

Light scattering measures large wavelength dielectric fluctuations. Even though $q a \ll 1$ can thus be assumed, the limit $q \rightarrow 0$ cannot be performed naively in Eq. (1). Because of density, momentum, and energy conservation, there are poles in the resolvent $R(z)=(\mathcal{L}-z)^{-1}$, which shift to vanishing frequency in this limit [15]. Using the Zwanzig-Mori formalism, these hydrodynamic low-frequency features can be identified. One introduces the reduced resolvent

$$
R^{\prime}(z)=Q \frac{1}{Q \mathcal{L} Q-z} Q
$$

where the projector $Q=1-P$ projects perpendicular to the hydrodynamic modes:

$$
P=\frac{\mid n(\mathbf{q}))(n(\mathbf{q}) \mid}{(n(\mathbf{q}) \mid n(\mathbf{q}))}+\frac{\mid \Theta(\mathbf{q}))(\Theta(\mathbf{q}) \mid}{(\Theta(\mathbf{q}) \mid \Theta(\mathbf{q}))}+\sum_{i} \frac{\left.\mid j_{i}(\mathbf{q})\right)\left(j_{i}(\mathbf{q}) \mid\right.}{\left(j_{i}(\mathbf{q}) \mid j_{i}(\mathbf{q})\right)}
$$

The standard hydrodynamic formulas are obtained in this approach if the reduced resolvent $R^{\prime}(z)$ is treated in a Markovian approximation, replacing its matrix elements with frequency-independent transport coefficients [15]. Generalized hydrodynamics differs from this by retaining the frequency dependence of $R^{\prime}(z)$ but still neglecting its wavevector dependence. This generalization is required for liquids at lower temperatures as the structural relaxation slows down strongly.

Following Ref. [14] we identify the fluctuating temperature $\Theta(\mathbf{q})$ with the kinetic-energy fluctuations, $e^{K}(\mathbf{q})$, that are orthogonal to the density fluctuations, $c_{V}^{0} \Theta(\mathbf{q})$ $=Q_{n} e^{K}(q)=e^{K}(\mathbf{q})-n(\mathbf{q})\left(e^{K} \mid n\right) /(n \mid n)$. Here $c_{V}^{0}=3 k_{B} / 2$ abbreviates the specific heat per particle of the kinetic degrees of freedom, and $Q_{n}$ is the projector orthogonal to the density. Conservation of the total energy $e(\mathbf{q})=e^{K}(\mathbf{q})$ $+e^{P}(\mathbf{q})$ implies

$$
c_{V}^{0} \mathcal{L} \Theta(\mathbf{q})=q j_{e}^{L}(\mathbf{q})-q j^{L}(\mathbf{q}) \frac{(e \mid n)}{(n \mid n)}-\mathcal{L} Q e^{P}(\mathbf{q}),
$$

where superscripts $L$ indicate the longitudinal part, $j^{L}$ $=\mathbf{q} \cdot \mathbf{j} / q, e^{P}(\mathbf{q})$ is the potential energy, and $\mathbf{j}_{e}(\mathbf{q})$ the totalenergy current. Note that since $\left(\Theta(\mathbf{q}) \mid e^{P}(\mathbf{q})\right)=0$ one can replace $Q_{n} e^{P}(\mathbf{q})=Q e^{P}(\mathbf{q})$. The hydrodynamic variables are orthogonal with normalizations: $(n(\mathbf{q}) \mid n(\mathbf{q}))=N S(q) / k_{B} T$, $\left(j_{k}(\mathbf{q}) \mid j_{l}(\mathbf{q})\right)=(N / m) \delta_{k l}$ and $(\Theta(\mathbf{q}) \mid \Theta(\mathbf{q}))=N T / c_{V}^{0}$. Here, $n$ is the average density of $N$ molecules, $m$ the molecular mass, and $S(q)$ is the equilibrium center-of-mass structure factor; $\delta_{k l}$ is Kronecker's symbol. Throughout the following we will neglect wave-vector dependencies caused by molecular length scales, and replace, e.g., the structure factor by its homogeneous limit given by the isothermal compressibility $\kappa_{T}: S(q)=S(0)+O\left((q a)^{2}\right)$ with $S(0)=n k_{B} T \kappa_{T}$.

Considering, in generalized hydrodynamics [16], the fluctuating temperature instead of energy fluctuations rests upon the experimental observation that the heat conduction of glasses and liquids is not drastically different. This aspect is discussed in Refs. [14,17] where the generalized hydrodynamics also is tested by molecular-dynamics simulations. This formulation of generalized hydrodynamics accounts straightforwardly for a frequency-dependent isochoric specific heat.

\section{Decomposition of dielectric fluctuations}

The exact resolvent calculus sketched in the previous section thus provides a reformulation of Eq. (1), see Eq. (A14) in Ref. [14]. The reduced dynamics $R^{\prime}(z)$ and the projector $P$, which projects onto the hydrodynamic variables, appear 


$$
\begin{aligned}
\left(\epsilon_{i j}(\mathbf{q})|R(z)| \epsilon_{k l}(\mathbf{q})\right)= & \left(\epsilon_{i j}(\mathbf{q})\left|R^{\prime}(z)\right| \epsilon_{k l}(\mathbf{q})\right)+\left(\epsilon_{i j}(\mathbf{q}) \mid\right. \\
& \times\left[1-R^{\prime}(z) \mathcal{L}\right] P R(z) P \\
& \left.\times\left[1-\mathcal{L} R^{\prime}(z)\right] \mid \epsilon_{k l}(\mathbf{q})\right) .
\end{aligned}
$$

Thus the coupling of the dielectric fluctuations to the hydrodynamic variables is found; explicitly it is given when writing out $P R(z) P$ in Eq. (5). Additionally there is a background spectrum, the first term on the right-hand side of Eq. (5).

Since the hydrodynamic modes have been projected out (generalized) hydrodynamics postulates that the limit $q \rightarrow 0$ in $R^{\prime}(z)$ can now be performed safely. This leads to the well-known result for Raman scattering [7]: The background spectrum consists of scalar, and symmetric-traceless-tensor scattering

$$
\begin{aligned}
\left(\epsilon_{i j}(\mathbf{q})\left|R^{\prime}(z)\right| \epsilon_{k l}(\mathbf{q})\right)= & \mathcal{S}(z) \delta_{i j} \delta_{k l}+\mathcal{T}(z)\left(\delta_{i k} \delta_{j l}+\delta_{i l} \delta_{j k}\right. \\
& \left.-\frac{2}{3} \delta_{i j} \delta_{k l}\right)+O\left(q^{2}\right)
\end{aligned}
$$

Explicit expression for $\mathcal{S}(z)$ and $\mathcal{T}(z)$ can be obtained by choosing special linear combinations of the dielectric tensor. Let $s_{00}=\left[\epsilon_{x x}+\epsilon_{y y}+\epsilon_{z z}\right] / 3$ denote the long-wavelength limit of the scalar part and $t_{20}=\left[2 \epsilon_{z z}-\epsilon_{x x}-\epsilon_{y y}\right] / \sqrt{12}$ the (helicity) $\nu=0$ component of the corresponding spherical tensor $t_{2 v}$ (the prefactors are conventional). Then, $\mathcal{S}(z)$ $=\left(s_{00}\left|R^{\prime}(z)\right| s_{00}\right)$ and $\mathcal{T}(z)=\left(t_{20}\left|R^{\prime}(z)\right| t_{20}\right)$ are the scalar and the tensor correlations. Let us state here explicitly that we assume that the long-wavelength static correlations of the dielectric tensor are characterized by two numbers only and are independent of the direction of $\mathbf{q} \rightarrow \mathbf{0}$.

Both spectral contributions $\mathcal{S}(z), \mathcal{T}(z)$ are autocorrelation functions of real variables with even time inversion symmetry. Their spectra thus are even and non-negative.

In order to simplify the discussion of the couplings to the hydrodynamic variables in Eq. (5), it is useful to consider the corresponding generalized constitutive equations $[14,15]$. These describe the temporal decay of the deviations in a variable, say in $\delta \epsilon_{i j}(\mathbf{q}, t)$, produced by an adiabatic perturbation with external fields coupling to the conserved variables, after the perturbations are switched off at time $t=0$ :

$$
\begin{aligned}
\left\langle\delta \epsilon_{i j}(\mathbf{q}, t)\right\rangle= & \sum_{\alpha}^{5}\left[\left\langle\delta A_{\alpha}(t)\right\rangle\left(A_{\alpha} \mid \epsilon_{i j}(\mathbf{q})\right) /\left(A_{\alpha} \mid A_{\alpha}\right)\right. \\
& -\int_{0}^{t} d \tau\left\langle\delta A_{\alpha}(\tau)\right\rangle\left(A_{\alpha} \mid i \mathcal{L} R^{\prime}(t\right. \\
& \left.\left.-\tau) \mid \epsilon_{i j}(\mathbf{q})\right) /\left(A_{\alpha} \mid A_{\alpha}\right)\right]
\end{aligned}
$$

where the (orthogonal) hydrodynamic variables are abbreviated: $A_{1}=n(\mathbf{q}), A_{2}=\Theta(\mathbf{q}), A_{3}=j_{x}(\mathbf{q}), A_{4}=j_{y}(\mathbf{q})$, and $A_{5}$ $=j_{z}(\mathbf{q})$. The special choice of the external perturbation considered in the constitutive equation, Eq. (7), prepares a fluctuation in $\left\langle\delta \epsilon_{i j}(\mathbf{q}, t)\right\rangle$, which decays slowly because it re- quires decay of fluctuations of the conserved variables at large wavelengths, $O(1 / q)$. Thus the time evolution of $\left\langle\delta \epsilon_{i j}(\mathbf{q}, t)\right\rangle$ in Eq. (7), is determined by time-dependent couplings to the generalized hydrodynamics of the distinct variables. As seen via a Laplace transformation, the identical couplings appear in Eq. (5) as in Eq. (7), expressing that via these couplings, close to equilibrium, dielectric fluctuations at long wavelengths acquire slow hydrodynamic components. Equation (5) further identifies the nonhydrodynamic components contributing to the background. The first term on the right-hand side of Eq. (7) describes static or instantaneous couplings, whereas the second term describes dynamic couplings that need time to build up and may be characterized by a finite response time. These will become important when approaching the glass transition upon cooling as then the structural relaxation times increase.

Density fluctuations are coupled to the dielectric fluctuations statically via the scalar scattering mechanism only, as follows from Eq. (7) when inserting the projector $P$ from Eq. (3):

$$
\left(n(\mathbf{q})\left|\left[1-\mathcal{L} R^{\prime}(z)\right]\right| \epsilon_{i j}(\mathbf{q})\right)=\left(n \mid s_{00}\right) \delta_{i j}+O\left(q^{2}\right) .
$$

The dynamic coupling vanishes because $\mathcal{L} n(\mathbf{q})=q_{k} j_{k}(\mathbf{q})$ is again a hydrodynamic variable. Furthermore the scalar density cannot couple to the dielectric tensor fluctuations $t_{2 \nu}$ in the limit $q \rightarrow 0$.

Similar arguments hold for the coupling of the temperature to the dielectric fluctuations. Since the kinetic energy is not conserved, there is a dynamic coupling in addition to the static one. In order to guarantee conservation of the total energy, Eq. (4) is used. Observing $\left(\mathcal{L} Q e^{P} \mid R^{\prime}(z)=\left(Q e^{P} \mid\right.\right.$ $+z\left(e^{P} \mid R^{\prime}(z)\right.$ and rearranging terms one finds to lowest order in $q$

$$
\begin{aligned}
& c_{V}^{0}\left(\Theta(\mathbf{q})\left|\left[1-\mathcal{L} R^{\prime}(z)\right]\right| \epsilon_{i j}(\mathbf{q})\right) \\
& \quad=\delta_{i j}\left\{\left(Q_{n} e \mid s_{00}\right)+z\left(e^{P}\left|R^{\prime}(z)\right| s_{00}\right)\right\}+O(q) .
\end{aligned}
$$

Further couplings of order $q$ can be ignored. The dynamic coupling in Eq. (9) arises from the separation of the totalenergy fluctuations into fast kinetic and (possibly) slow potential ones. Thus the simplified handling of the reduced resolvent in this generalized hydrodynamics has to be paid by an additional frequency-dependent coupling to temperature fluctuations.

Finally for the coupling of dielectric fluctuations to current fluctuations

$$
\begin{aligned}
& \left(j_{k}(\mathbf{q})\left|\left[1-\mathcal{L} R^{\prime}(z)\right]\right| \boldsymbol{\epsilon}_{i j}(\mathbf{q})\right) \\
& \quad=-\sum_{l} q_{l}\left(\tau_{k l}(\mathbf{q})\left|R^{\prime}(z)\right| \epsilon_{i j}(\mathbf{q})\right) / m,
\end{aligned}
$$

one finds a purely dynamical coupling, as first recognized within so-called two variable models [2,3]. A static coupling is excluded, since the currents $j_{k}$ and the dielectric tensor $\epsilon_{i j}$ have different time-reversal symmetry. The dynamic coupling to the stress tensor, $\tau_{k l}$, which appears because of mo- 
mentum conservation, $\mathcal{L}_{k}(\mathbf{q})=\Sigma_{l} q_{l} \tau_{k l}(\mathbf{q}) / m$, can be evaluated in the long-wavelength limit:

$$
\begin{aligned}
\left(\tau_{k l}(\mathbf{q})\left|R^{\prime}(z)\right| \epsilon_{i j}(\mathbf{q})\right) \\
=\left(p\left|R^{\prime}(z)\right| s_{00}\right) \delta_{i j} \delta_{k l}+\left(\tau_{20}\left|R^{\prime}(z)\right| t_{20}\right) \\
\quad \times\left[\delta_{i k} \delta_{j l}+\delta_{i l} \delta_{j k}-\frac{2}{3} \delta_{i j} \delta_{k l}\right]+\mathcal{O}\left(q^{2}\right) .
\end{aligned}
$$

Here $p=\left[\tau_{x x}+\tau_{y y}+\tau_{z z}\right] / 3$ and $\tau_{20}=\left[2 \tau_{z z}-\tau_{x x}-\tau_{y y}\right] / \sqrt{12}$ denote the long-wavelength limits of the pressure and of the transversal stress tensor.

According to the basic assumption of (generalized) hydrodynamics $[14,15]$, one has to keep terms only to the order indicated, the remaining ones are assumed to be regular with respect to frequency $z$ in the limit $q \rightarrow 0$.

\section{RESULTS}

From Eqs. (5) to (11) the light-scattering spectra follow if a scattering geometry is chosen and the appropriate tensor elements are calculated; see Appendix A for the used geometry. Polarizations vertical to $V$, or in the scattering plane $H$, are considered, where the standard abbreviation $I_{i o}$ denotes polarizations for incoming and outgoing light. The spectra depend on $q, \omega$ and scattering angle $\theta$. We find $I_{H V}(q, \theta, \omega)=I_{V H}(q, \theta, \omega)$ as predicted by Rayleigh's reciprocity theorem [1].

\section{A. Total scattered intensities}

The total scattered intensities (except for standard coefficients [1]) can be obtained directly from Eq. (A4) in Appendix A and consist of scalar and tensor scattering [7]:

$$
\begin{gathered}
I_{V V}(q, \theta)=\left(s_{00} \mid s_{00}\right)+\frac{4}{3}\left(t_{20} \mid t_{20}\right), \\
I_{V H}(q, \theta)=\left(t_{20} \mid t_{20}\right), \\
I_{H H}(q, \theta)=\cos ^{2} \theta\left(s_{00} \mid s_{00}\right)+\left(1+\frac{1}{3} \cos ^{2} \theta\right)\left(t_{20} \mid t_{20}\right) .
\end{gathered}
$$

The intensities are wave-vector independent as the limit $q a$ $\ll 1$ is considered in systems where all molecular correlations are short ranged. The conservation laws affect the spectral shapes only, i.e. cause low-lying hydrodynamic lines, but do not lead to long-ranged static correlations. Concurrently, the static couplings of the conserved variables to the dielectric fluctuations in Eqs. (8) to (10) are $q$ independent for small wave numbers. See Sec. IV, for why these results appear violated when considering the hydrodynamic limits.

\section{B. Depolarized spectrum}

Using the decomposition of the off-diagonal dielectric fluctuations, Eqs. (7), (10), and (11), one can identify a dynamic coefficient, $a_{V H}(z)$, which describes the coupling to the generalized hydrodynamic variables:

$$
a_{V H}(t)=\frac{1}{N}\left(\tau_{20}\left|R^{\prime}(t)\right| t_{20}\right) .
$$

It is a generalized elasto-optic or Pockels' constant familiar from light scattering in solids [7], and is a real and symmetric function of time, as the two tensor variables determining it are real with even time parity. Its Laplace transform $a_{V H}(z)$, therefore has an even spectrum $a_{V H}^{\prime \prime}(\omega)$. This Pockels' constant describes the dynamic coupling of the transverse current into the dielectric fluctuations and the constitutive equation becomes

$$
\left\langle\delta \epsilon_{V H}(\mathbf{q}, t)\right\rangle=-i q \cos \frac{\theta}{2} \int_{0}^{t} d \tau a_{V H}(t-\tau)\left\langle\delta j_{y}(\mathbf{q}, \tau)\right\rangle .
$$

From Eqs. (5) and (6) follows the general result for the depolarized spectrum:

$$
\left(\epsilon_{V H}(\mathbf{q}, z) \mid \epsilon_{V H}(\mathbf{q})\right)=\mathcal{T}(z)+q^{2} \cos ^{2} \frac{\theta}{2} a_{V H}(z)^{2} C_{j j}^{T}(q, z) .
$$

Here $C_{j j}^{T}(q, z)=\left(j^{T}(q, z) \mid j^{T}(q)\right)$ denotes the correlation function of the transversal current fluctuations. The spectrum consists of a background arising from the symmetric scattering in Eq. (6), which commonly is discussed as a Raman line, and of couplings to the current fluctuations. Naively evaluating the depolarized spectrum at vanishing wave vector would neglect this additional contribution. It is small, of order $\mathcal{O}\left(q^{2}\right)$, but is characterized by a time scale that diverges in the hydrodynamic limit $q \rightarrow 0$, and therefore dominates the low-frequency spectrum. The full current correlators appear in Eq. (17), which stresses that no assumptions about translational-rotational coupling are required in order to derive Eq. (17). Explicitly this has been shown by the derivation of Eq. (17) for a liquid of spherical particles in Ref. [19], which was tested in a simulation [20].

\section{Polarized spectra}

For the $V V$ spectrum, Eq. (11) suggests to introduce the elasto-optical constant

$$
a_{V V}(t)=\frac{2}{3} a_{V H}(t)-\frac{\left(p\left|R^{\prime}(t)\right| s_{00}\right)}{N} .
$$

It has identical properties as $a_{V H}(t)$ since the tensor variables entering its definition again are real with even time parity. Another time-dependent coupling function arises from the temperature fluctuations as described in Eq. (9):

$$
\xi(z)=\xi+z \frac{\left(e^{P}\left|R^{\prime}(z)\right| s_{00}\right)}{N T},
$$

where the thermodynamic derivative $\xi=\left(\partial s_{00} / \partial T\right)_{n} / n$ $=\left(Q_{n} e \mid s_{00}\right) /(N T)$ is written, which contains the total energy perpendicular to density fluctuations [14]. Clearly, Eq. (19) presents a generalized time- or frequency-dependent thermodynamic derivative. The scalar variables entering its time or 
frequency-dependent term are real with even time parity, and $\xi^{\prime \prime}(\omega) / \omega$ consequently is an even function of $\omega$. The constitutive equation coupling the fluctuating hydrodynamic variables into $\delta \epsilon_{V V}(\mathbf{q}, t)$, from Eq. (7), becomes

$$
\begin{aligned}
\left\langle\delta \epsilon_{V V}(\mathbf{q}, z)\right\rangle= & \left(\partial s_{00} / \partial n\right)_{T}\langle\delta n(\mathbf{q}, z)\rangle+\xi(z)\langle\delta \Theta(\mathbf{q}, z)\rangle \\
& +a_{V V}(z) q\left\langle\delta j^{L}(\mathbf{q}, z)\right\rangle,
\end{aligned}
$$

where the thermodynamic relation $\left(n \mid s_{00}\right)$ $=N n \kappa_{T}\left(\partial s_{00} / \partial n\right)_{T}$ is used. Collecting the terms in Eq. (5) one obtains when applying the mass-conservation law, which gives $q^{2} C_{j j}^{L}(q, z)=q z C_{n j}^{L}(q, z)=z^{2} C_{n n}(q, z)+z N n \kappa_{T}$ and $q C_{\Theta j}^{L}(q, z)={ }_{z} C_{\Theta n}(q, z)$,

$$
\begin{aligned}
\left(\epsilon_{V V}(\mathbf{q}, z) \mid \epsilon_{V V}(\mathbf{q})\right)= & \mathcal{S}(z)+4 \mathcal{T}(z) / 3+z a_{V V}^{2}(z) N n \kappa_{T} \\
& +2\left(\partial s_{00} / \partial n\right)_{T} a_{V V}(z) N n \kappa_{T} \\
& +\left[\left(\partial s_{00} / \partial n\right)_{T}+z a_{V V}(z)\right]^{2} C_{n n}(q, z) \\
& +\xi(z)^{2} C_{\Theta \Theta}(q, z)+2\left[\left(\partial s_{00} / \partial n\right)_{T}\right. \\
& \left.+z a_{V V}(z)\right] \xi(z) C_{n \Theta}(q, z) .
\end{aligned}
$$

Here $C_{n n}(q, z)$ denotes the density-density correlation function, $C_{\Theta \Theta}(q, z)$ the temperature-temperature correlation function, etc; see Appendix B. Equation (21) is our principal result for the $V V$ spectrum. It extends the conventional hydrodynamic spectra to arbitrary frequencies. The smallwave-vector singularities are encoded in the generalized hydrodynamic correlation functions $C_{\alpha \beta}(q, z)$, which are determined by the true resolvent $R(z)$, and can thus e.g., be obtained from simulations.

From the information on the depolarized and the polarized spectrum also the $I_{H H}$ spectrum can be obtained even though it is not a simple linear combination. The fluctuating variable coupling to the distinct variables for the $H H$ scattering in the geometry of Appendix A is given by

$$
\begin{aligned}
\left\langle\delta \epsilon_{H H}(\mathbf{q}, z)\right\rangle= & -\cos \theta\left(\partial s_{00} / \partial n\right)_{T}\langle\delta n(\mathbf{q}, z)\rangle-\cos \theta \xi(z) \\
& \times\langle\delta \Theta(\mathbf{q}, z)\rangle-\left[a_{H H}(z) \cos \theta\right. \\
& \left.-a_{V H}(z)\right] q\left\langle\delta j^{L}(\mathbf{q}, z)\right\rangle
\end{aligned}
$$

where we abbreviated $a_{H H}(z)=a_{V V}(z)-a_{V H}(z)$. The general spectrum in the $H H$ geometry now reads

$$
\begin{aligned}
\left(\epsilon_{H H}(\mathbf{q}, z) \mid \boldsymbol{\epsilon}_{H H}(\mathbf{q})\right)= & \left.\mathcal{S}(z) \cos ^{2} \theta+\mathcal{T}(z)\left(1+\frac{1}{3} \cos ^{2} \theta\right)+z\left[a_{H H}(z) \cos \theta-a_{V H}(z)\right]^{2} N n \kappa_{T}+2 \frac{\partial s_{00}}{\partial n}\right)_{T} \cos \theta\left[a_{H H}(z) \cos \theta\right. \\
& \left.\left.-a_{V H}(z)\right] N n \kappa_{T}+\left\{\left[\frac{\partial s_{00}}{\partial n}\right)_{T}+z a_{H H}(z)\right] \cos \theta-z a_{V H}(z)\right\}^{2} C_{n n}(q, z)+\xi(z)^{2} \cos ^{2} \theta C_{\Theta \Theta}(q, z) \\
& \left.+2 \xi(z) \cos \theta\left\{\left[\frac{\partial s_{00}}{\partial n}\right)_{T}+z a_{H H}(z)\right] \cos \theta-z a_{V H}(z)\right\} C_{n \Theta}(q, z)
\end{aligned}
$$

One notices that even for a general molecular fluid, transverse current fluctuations do not couple into the $\mathrm{HH}$ spectrum.

\section{Generalized Green-Kubo relations}

Ten frequency-dependent matrix elements built with the reduced resolvent $R^{\prime}(z)$ have been identified in the expressions for the light-scattering spectra in supercooled liquids. Five generalized transport coefficients and thermodynamic derivatives are needed in order to describe the correlators of the hydrodynamic variables [14]. They are the shear viscosity $K_{s}(z)$, the thermal conductivity $\lambda(z)$, the dynamic specific heat $c_{V}(z)$, the dynamic tension coefficient $\beta(z)$, and the longitudinal stress relaxation kernel $K_{l}(z)$ (explicit expressions are summarized in Appendix B). The remaining five frequency-dependent kernels encode the details of the light-scattering process: $\mathcal{S}(z), \mathcal{T}(z), a_{V H}(z), a_{V V}(z)$, and $\xi(z)$.

These expressions involving reduced resolvents are very suitable for approximations, since they do not exhibit hydrodynamic singularities. In order to determine them from other theoretical approaches or from computer simulations, it is, however, more convenient to find a formulation in terms of correlation functions involving the full dynamics. For the considered cases at $q=0$, this is made possible by the conservation laws that allow one to derive Green-Kubo relations for the memory functions or transport coefficients expressing them in terms of autocorrelation functions of the corresponding fluxes or time integrals thereof $[15,16]$. From the identity [14]:

$$
\begin{aligned}
\left(\tilde{X}\left|R^{\prime}(z)\right| \tilde{Y}\right)= & (\tilde{X}|R(z)| \tilde{Y})+\left(\tilde{X} \mid\left[1-R^{\prime}(z) \mathcal{L}\right]\right. \\
& \left.\times P R(z) P\left[\mathcal{L} R^{\prime}(z)-1\right] \mid \widetilde{Y}\right),
\end{aligned}
$$

one observes that the reduced matrix elements can be rewritten as full matrix elements and correlation functions of the hydrodynamic variables contained in $P R(z) P$ with frequency-dependent coefficients. Since for $q \rightarrow 0$ the coefficients involving $\mathcal{L} n_{q}$ and $\mathcal{L} j_{q}$ vanish due to particle and momentum conservation, only the temperature fluctuations contribute to the frequency dependence of the coefficients. To derive a generalized Green-Kubo relation, we only need variables $\widetilde{X}=Q X$ and $\widetilde{Y}=Q Y$, respectively. Therefore all static 
couplings $\tilde{X} \mid P R(z) P$ to the hydrodynamic variables in Eq. (24) will vanish too and the generalized Green-Kubo relation is given by

$$
\left(X\left|R^{\prime}(z)\right| Y\right)=(Q X|R(z)| Q Y)+\frac{\left\{\left(X \mid Q e^{P}\right)+z\left(X\left|R^{\prime}(z)\right| e^{P}\right)\right\}\left\{\left(Q e^{P} \mid Y\right)+z\left(e^{P}\left|R^{\prime}(z)\right| Y\right)\right\}}{N T z c_{V}(z)} \quad \text { for } q \rightarrow 0 .
$$

Here we made use of Eqs. (4) and (B3) in the limit $q \rightarrow 0$ and of the identity $R^{\prime}(z) \mathcal{L} Q=Q+z R^{\prime}(z)$. Rotational invariance implies that the second term in Eq. (25) is nonzero only for scalar variables $X, Y$. Thus, e.g., the standard Green-Kubo relations for the shear viscosity, $X=Y=\tau_{20}$, and heat diffusion, $X=Y=j_{e}^{L}$, are found. Moreover, it follows that in the elasto-optic constant $a_{V H}(z)$, Eq. (15), and in the tensor background spectrum $\mathcal{T}(z)$, Eq. (6), the reduced resolvent can be replaced with the full dynamics.

In order to obtain tractable expressions for the remaining kernels and make contact with the standard Kadanoff-Martin approach $[16,18]$, we define another, the conventional, fluctuating temperature by $c_{V} \widetilde{T}(\mathbf{q})=e(\mathbf{q})-n(\mathbf{q})(e \mid n) /(n \mid n)$, with normalization $(\widetilde{T}(\mathbf{q}) \mid \widetilde{T}(\mathbf{q}))=N T / c_{V}$. Also, let $\widetilde{Q}$ denote the projector orthogonal to density, currents, and $\widetilde{T}(\mathbf{q})$. Choosing $X=Y=e^{P}$ in Eq. (25) one finds

$$
\frac{\left(Q e^{P}(z) \mid Q e^{P}\right)}{N T}=\frac{c_{V}(z)-c_{V}}{z}-\frac{\left[c_{V}(z)-c_{V}^{0}\right]^{2}}{z c_{V}(z)} .
$$

Since $Q e^{P}=\widetilde{Q} e^{P}+\left(c_{V}-c_{V}^{0}\right) \widetilde{T}$ the left-hand side implicitly contains hydrodynamic poles due to energy conservation. However,

$$
\frac{\left(Q e^{P}(z) \mid Q e^{P}\right)}{N T}=\frac{\left(\widetilde{Q} e^{P}(z) \mid \widetilde{Q} e^{P}\right)}{N T}-\frac{\left(c_{V}-c_{V}^{0}\right)^{2}}{z c_{V}},
$$

and, according to Kadanoff-Martin, the first term on the right-hand side is free of poles in the limit $z \rightarrow 0$ [18]. Combining Eqs. (26) and (27) one derives

$$
c_{V}(z)=c_{V} \frac{\left(c_{V}^{0}\right)^{2}}{\left(c_{V}^{0}\right)^{2}-z c_{V}\left(\widetilde{Q} e^{P}(z) \mid \widetilde{Q} e^{P}\right) /(N T)} .
$$

In the liquid phase the dynamic specific heat attains its thermodynamic value $c_{V}(z) \rightarrow c_{V}$ for $z \rightarrow 0$. Equation (28) demonstrates explicitly that the Götze-Latz resolvent $R^{\prime}(z)$ indeed is devoid of all hydrodynamic singularities and is compatible with the conventional Kadanoff-Martin formalism. It differs in an explicit frequency dependence of $c_{V}(z)$, which arises from the splitting of the conventional temperature fluctuations $\widetilde{T}(q, t)$ into fast, kinetic ones $\Theta(q, t)$ and structural slow ones.

Similarly, substituting $X=p$ and $Y=e^{P}$ in Eq. (25) yields

$$
\frac{\left(Q p(z) \mid Q e^{P}\right)}{N m T}=\frac{\beta(z)-\beta}{z}-\frac{\left[\beta(z)-\beta^{0}\right]\left[c_{V}(z)-c_{V}^{0}\right]}{z c_{V}(z)},
$$

where $\quad \beta^{0}=\left(p \mid Q_{n} e^{K}\right) /(N m T)$. Since $\quad Q p=\widetilde{Q} p$ $+m \beta^{0} \widetilde{Q} e^{P} / c_{V}^{0}+m\left(\beta-\beta^{0}\right) \widetilde{T}$, the left-hand side can be written as

$$
\begin{aligned}
\frac{1}{N m T}\left(Q p(z) \mid Q e^{P}\right)= & \frac{1}{N m T}\left[\widetilde{Q} p(z) \mid \widetilde{Q} e^{P}\right] \\
& +\frac{\beta^{0}}{N T c_{V}^{0}}\left(\widetilde{Q} e^{P}(z) \mid \widetilde{Q} e^{P}\right) \\
& -\frac{\left(\beta-\beta^{0}\right)\left(c_{V}-c_{V}^{0}\right)}{z c_{V}} .
\end{aligned}
$$

Again the memory kernels appearing on the right-hand side are regular in the low-frequency limit. Collecting terms leads to the generalized Green-Kubo formula for the dynamic tension coefficient

$$
\beta(z)=\beta \frac{c_{V}(z)}{c_{V}}+\frac{c_{V}(z)}{c_{V}^{0}} z \frac{\left(\widetilde{Q} p(z) \mid \widetilde{Q} e^{P}\right)}{N m T} .
$$

In a similar fashion the corresponding Green-Kubo formulas for the dynamic temperature coupling, the scalar background spectrum, the remaining term contributing to $a_{V V}(z)$, and the longitudinal stress-stress correlation function can be obtained:

$$
\begin{gathered}
\xi(z)=\xi \frac{c_{V}(z)}{c_{V}}+\frac{c_{V}(z)}{c_{V}^{0}} z \frac{\left(\widetilde{Q} s_{00}(z) \mid \widetilde{Q} e^{P}\right)}{N T} \\
\mathcal{S}(z)=\frac{N T \xi(z)^{2}}{z c_{V}(z)}-\frac{N T}{z c_{V}} \xi^{2}+\left(\widetilde{Q} s_{00}(z) \mid \widetilde{Q} s_{00}\right) \\
\frac{\left(p\left|R^{\prime}(z)\right| s_{00}\right)}{N}=\frac{m \beta(z) T}{z c_{V}(z)} \xi(z)-\frac{m \beta T}{z c_{V}} \xi+\frac{\left(\widetilde{Q} p(z) \mid \widetilde{Q} s_{00}\right)}{N} \\
\frac{K_{l}(z)}{n m}=\frac{m T}{z c_{V}(z)} \beta(z)^{2}-\frac{m T}{z c_{V}} \beta^{2}+\frac{\left(\widetilde{Q} \tau^{L}(z) \mid \widetilde{Q} \tau^{L}\right)}{N m}
\end{gathered}
$$

In particular, the last term on the right-hand side of Eq. (35) is related to the longitudinal viscosity $i \eta_{l}=\left(\widetilde{Q} \tau^{L}(z\right.$ $\left.\rightarrow 0) \mid \widetilde{Q} \tau^{L}\right) n / N$. Therefore in the low-frequency limit where $c_{V}(z)=c_{V}+i z c_{V}^{\prime \prime}$, and $\beta(z)=\beta+i z \beta^{\prime \prime}$ one finds

$$
\frac{K_{l}(z \rightarrow 0)}{n m}=-i m T \beta^{2} \frac{c_{V}^{\prime \prime}}{c_{V}^{2}}+2 i m T \beta \frac{\beta^{\prime \prime}}{c_{V}}+i \frac{\eta_{l}}{n m} .
$$


To summarize, all ten frequency-dependent kernels can be expressed in terms of the full resolvent $R(z)$, and therefore they can be obtained directly from molecular-dynamics simulations.

\section{DISCUSSION}

\section{A. Depolarized spectra}

The depolarized frequency-dependent spectrum, Eq. (17), shall be discussed in detail as it provides the most compact expression but also exhibits clear qualitative changes when supercooling the liquid. It consists of three frequencydependent contributions, a background, the Pockels constant, and the transverse current correlator.

The current correlators can be taken from theories for the dynamics of the liquid under study or from computer simulations. Alternatively, the generalized hydrodynamic approach shifts the problem of calculating the transverse correlator to the problem of calculating correlations of the transversal-stress tensor, namely, the frequency-dependent shear modulus, $K_{s}(z)=\left(\tau_{20}\left|R^{\prime}(z)\right| \tau_{20}\right) n / N$, where the resolvent devoid of hydrodynamic fluctuations from Eq. (2) appears again. The separation of the hydrodynamic poles from structural relaxation thus is achieved leading to [14]:

$$
C_{j j}^{T}(q, z)=\frac{-(N / m)}{z+q^{2} K_{s}(z) / n m}
$$

The result from hydrodynamic theory for the depolarized light scattering from equilibrium molecular liquids can be obtained if the frequency dependence of memory functions built with the reduced resolvent $R^{\prime}(z)$ is neglected by using the Markovian low-frequency limit. Then the depolarized Pockels' constant becomes purely imaginary:

$$
a_{V H}(z) \rightarrow i a_{V H}^{\prime \prime}=i \int_{0}^{\infty} d t a_{V H}(t) \text { for } z \rightarrow 0 \text {. }
$$

Therefore, and using the standard hydrodynamic result for the shear viscosity, $K_{s}(z \rightarrow 0) \rightarrow i \eta_{s}$, one recovers the result for the depolarized spectrum first obtained within simple models in Refs. [2,3]:

$$
\begin{aligned}
I_{V H}(q, \theta, \omega)_{\text {hy. l. }}= & \mathcal{T}^{\prime \prime}(\omega=0)-q^{2}\left(a_{V H}^{\prime \prime}\right)^{2} \cos ^{2} \frac{\theta}{2} \\
& \times \frac{N q^{2} \eta_{s} /\left(n m^{2}\right)}{\omega^{2}+\left(q^{2} \eta_{s} / n m\right)^{2}} .
\end{aligned}
$$

The transverse momentum diffusion cuts a central line of half-width $q^{2} \eta_{s} /(m n)$ and amplitude proportional to $\left(a_{V H}^{\prime \prime} \cos \theta / 2\right)^{2} n N / \eta_{s}$ out of a flat background. Note, that within hydrodynamics the background is structureless, and that the spectrum is positive owing to an elementary Schwartz inequality, $\left(t_{20} \mid t_{20}\right)\left(\tau_{20} \mid \tau_{20}\right) \geqslant\left(\tau_{20} \mid t_{20}\right)^{2}$.

The structural relaxation of liquids cooled down to and below the melting temperature, slows down strongly, and the frequency dependence of the memory functions with reduced resolvent, can no longer be neglected; for a discussion of structural relaxation, see e.g., the review [21]. The result in Eq. (17) can handle this situation, as the memory functions built with $R^{\prime}(z)$ - there are 3 in Eqs. (17) and (37) - may either be modeled appropriately or can be taken from other theories or simulations. Within the generalized hydrodynamic approach, a glass or amorphous solid is obtained whenever a structural relaxation process, with time scale $\tau_{\alpha}$, is slow compared to the hydrodynamic frequencies. Assuming further that the dynamics in $R^{\prime}(t)$ at shorter times, denoted by $\tau_{\beta}$, can be neglected for this frequency range, then the memory functions in Eq. (17) can be approximated by

$$
K_{s}(z) \rightarrow \frac{-G_{\infty}}{z}+i \Gamma_{s}, \quad a_{V H}(z) \rightarrow \frac{-a_{V H}^{\infty}}{z},
$$

for $1 / \tau_{\alpha} \ll|z| \ll 1 / \tau_{\beta}$. This is equivalent to time-independent values, $K_{s}(t)=G_{\infty}$ and $a_{V H}(t)=a_{V H}^{\infty}$ for $\tau_{\beta} \ll t \ll \tau_{\alpha}$. Therefore, the poles in Eq. (40) are called nonergodicity poles as they describe frozen-in, nonrelaxing components. $G_{\infty}=m n c_{T}^{2}$ is the glassy shear modulus familiar from Maxwell's model and $a_{H V}^{\infty}$ is the Pockels' constant (often denoted $\mathcal{P}_{44}$ ) quantifying the elasto-optic coupling in the glass [7]. Whereas $G_{\infty}$ and $\Gamma_{s}$ need to be positive, the sign of $a_{H V}^{\infty}$ is undetermined; a next-to-leading imaginary part in $a_{H V}$ exists in principle but does not contribute to the spectrum in the hydrodynamic limit. Equations (17) and (40) predict for the hydrodynamic glass spectrum:

$$
\begin{aligned}
I_{V H}(q, \theta, \omega)_{\text {hy g g }}= & \mathcal{T}_{g}^{\prime \prime}+\left(q \cos \frac{\theta}{2} a_{V H}^{\infty}\right)^{2} \\
& \times \frac{q^{2} \Gamma_{s} /(m n)}{\left(\omega^{2}-q^{2} c_{T}^{2}\right)^{2}+\left(\omega q^{2} \Gamma_{s} / n m\right)^{2}}
\end{aligned}
$$

Two transverse phonon peaks characterized by the transverse sound velocity $c_{T}$, and a width $\propto q^{2} \Gamma_{s}$, appear, which are described as damped harmonic oscillations. The background consists of a central line, which cannot be resolved and a structureless continuum $\mathcal{T}(z)=-\mathcal{T}_{\infty} / z+i \mathcal{T}_{g}^{\prime \prime}$.

Note, that both hydrodynamic expressions, Eqs. (39) and (41), do not fulfill the sum rule for the total intensity, Eq. (13), and imply wave-vector-dependent total scattered intensities. The reasons, of course, are the Markovian approximations in Eqs. (17) and (40), which are restricted to describe the dynamics in the hydrodynamic range. Nontrivial spectra obtained in glasses on frequency scales characterized by $\tau_{\beta}$ [22] also require more elaborate expressions for the memory functions in Eq. (17).

For temperatures around the liquid-to-glass crossover at $T_{c}$, neither the assumption $\tau_{\alpha} \omega \gg 1$, nor the estimate $\tau_{\beta} \omega$ $\ll 1$ hold and the depolarized spectra exhibit anomalies [23]. The mode coupling theory of the structural relaxation there suggests modeling the reduced resolvent as $K_{s}(z) \approx-G_{\infty}[1$

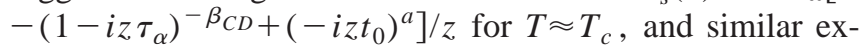
pressions for the other two memory functions. Whereas the Cole-Davidson behavior (the first two terms) is a (rough) 
model of the $\alpha$ process, and $\beta_{C D}$-as well as $\tau_{\alpha}$-will differ for different resolvent matrix elements, the power law with exponent $a$ describes the universal "critical" decay close to the transition. Here $t_{0}$ is a microscopic time and the exponent $a$ and the (true, universal) exponent $b$ of the high-frequency von Schweidler wing of the $\alpha$ process, $K_{s}\left(1 / \tau_{\alpha} \ll z \ll 1 / \tau_{\beta}\right)$ $\sim\left(-i z \tau_{\alpha}\right)^{-b} / z$, are related; see e.g., the review [21] for further information.

\section{B. Polarized spectra}

The most prominent feature of the polarized spectrum, the Brillouin peaks, arise from propagating sound waves. Upon cooling the liquid, structural relaxation manifests itself predominately by a gradual change of the sound velocity and the damping constant. Considering the enormous increase of the transport coefficients, e.g., the longitudinal viscosity that describes the damping in the liquid, this clearly points out the necessity to consider the frequency dependence of the reduced resolvent as done in generalized hydrodynamics. Furthermore, the couplings $a_{V V}(z), \xi(z)$ as well as the background spectra $\mathcal{S}(z), \mathcal{T}(z)$ exhibit nontrivial $z$ dependencies in the frequency regime of interest.

The structural relaxation of the Pockels' constant $a_{V V}(z)$ (with numerically different constants) can be modeled as given in Eqs. (38) and (40) and as described at the end of the previous section. The explicit factor $z$ in the frequencydependent part in $\xi(z)$, Eq. (19), cancels a possible nonergodicity pole. The coupling to temperature fluctuations interpolates smoothly between its low-frequency thermodynamic value $\left(\partial s_{00} / \partial T\right)_{n}$ and a high-frequency coupling, $\xi_{\infty}$, characteristic for a glass. A renormalization also appears in the effective coupling to the density fluctuations, which in Eq. (21) is described by the Pockels' constant $\mathcal{P}_{12}=\left(\partial s_{00} / \partial n\right)_{T}$ $+z a_{V V}(z)$ and in Eq. (23) by $\left(\mathcal{P}_{12} \cos \theta-\mathcal{P}_{44}\right)$, respectively, where $\mathcal{P}_{44}=z a_{V H}(z)$. Note, that the frequency-dependent renormalization of $\left(\partial s_{00} / \partial T\right)_{n}$ in Eq. (19) vanishes, if only the hydrodynamic fluctuations, density and temperature, contribute to the scalar scattering; then also $a_{V V}(z)=\frac{2}{3} a_{H V}(z)$.

In order to obtain the spectrum in the true hydrodynamic limit one substitutes the appropriate correlation functionssee Appendix B-and replaces all memory functions with their low-frequency limits; only in $c_{V}(z), \beta(z)$, and $\xi(z)$ linear terms in $z$ need to be kept as can be seen from Eqs. (28), (31), and (32). There are the three familiar hydrodynamic resonances superimposed on the Raman background: the Brillouin doublet of sound modes and the Rayleigh heat pole. The spectrum is obtained from determining the residues of these poles to lowest order in frequency and wave vector.

$$
\begin{aligned}
I_{V V}(q, \theta, \omega)_{h y . l .}= & \left(\widetilde{Q} s_{00}(\omega=0) \mid \widetilde{Q}_{00}\right)^{\prime \prime}+4 \mathcal{T}^{\prime \prime}(\omega=0) / 3+N n \kappa_{T} \mathcal{P}_{12}^{2}\left[\frac{X^{\mathrm{Br}}}{\gamma} \frac{c^{2} q^{4} \Gamma_{l}}{\left(\omega^{2}-c^{2} q^{2}\right)^{2}+\left(\omega q^{2} \Gamma_{l}\right)^{2}}\right. \\
& \left.+X^{\mathrm{R}} \frac{\gamma-1}{\gamma} \frac{q^{2} D_{T}}{\omega^{2}+\left(q^{2} D_{T}\right)^{2}}\right],
\end{aligned}
$$

where the adiabatic sound velocity $c=\sqrt{\gamma /\left(m n \kappa_{T}\right)}$, the longitudinal sound damping $\Gamma_{l}=D_{T}(\gamma-1)+\eta_{l} /(m n)$, and the heat diffusion constant $D_{T}=\lambda " / c_{P}$ appear (see Appendix B for details). The Pockels constant is given by the thermodynamic derivative, $\left.\mathcal{P}_{12}=\partial s_{00} / \partial n\right)_{T}$, and the flat background consists of scalar and tensor parts. Neglecting contributions from temperature fluctuations, $X^{\mathrm{R}}=X^{\mathrm{Br}}=1$, one regains the well-known result for light scattering from (hydrodynamic) density fluctuations $[1,7,15]$. Then, the Landau-Placzek result $(\gamma-1)$ is recovered for the relative intensity of the Rayleigh to the Brillouin lines, where $\gamma=c_{P} / c_{V}$ denotes the ratio of the isobaric to isochoric heat capacity $[1,7,15]$. In the general case, scattering from temperature fluctuations leads to (presumably small) corrections: $X^{\mathrm{R}}=\left[1-\left(c_{0}^{2} / \beta\right)\right.$ $\left.\times\left(\xi / \mathcal{P}_{12}\right)\right]^{2}$ and $X^{\mathrm{Br}}=\left[1+(\gamma-1)\left(c_{0}^{2} / \beta\right)\left(\xi / \mathcal{P}_{12}\right)\right]^{2}$.

For the glass, $\omega \tau_{\alpha} \gg 1$, actually the identical formula holds where, however, the isothermal compressibility, the sound velocity and damping constants, as well as the couplings and the background - compare Eqs. (39) and (41) are renormalized. The high-frequency values of the memory functions appear in the formally identical definitions of $c$, $D_{T}$, and $\Gamma_{l}$, where, in the frequency window $1 / \tau_{\alpha} \ll \omega$ $\ll 1 / \tau_{\beta}$, simple Markovian expressions like $c_{V}(z)=c_{V}^{\infty}$ $+i z c_{V, g}^{\prime \prime}$ are appropriate for ergodic matrix elements $\left[c_{V}(z)\right.$, $\beta(z), \xi(z)$, and $\lambda(z)]$, and frozen-in components, leading to $K_{l}(z)=-K_{l}^{\infty} / z+i K_{l, g}^{\prime \prime}$, appear in $K_{l}(z), a_{V V}(z)$, and in the Raman background lines. Thus, e.g., the expression for the Pockels constant becomes: $\left.\mathcal{P}_{12}^{\infty}=\partial s_{00} / \partial n\right)_{T}-a_{V V}^{\infty}$. A nontrivial renormalization appears in the isothermal sound velocity or equivalently the isothermal compressibility, because of the frozen structural relaxation in the longitudinal friction function:

$$
\left(c_{T}^{\infty}\right)^{2}=\frac{1}{m n \kappa_{T}^{\infty}}=c_{0}^{2}+\frac{K_{l}^{\infty}}{m n}
$$

as first observed by Mountain [6] and predicted from microscopic expressions by the mode-coupling theory [21]. The glass is less compressible than the corresponding liquid. Thus the glass sound velocity is $c^{\infty}=\sqrt{\gamma^{\infty}} c_{T}^{\infty}$ $=\sqrt{\left(c_{T}^{\infty}\right)^{2}+m T\left(\beta^{\infty}\right)^{2} / c_{V}^{\infty}}$. Note that the reduction of the Brillouin and Rayleigh intensities described by Eqs. (42) and (43) is caused by the appearance of frozen-in density fluc- 
tuations (an elastic line called the Mountain line) that contribute the missing weight, $N n \kappa_{T}\left[\left(\partial s_{00} / \partial n\right)_{T}^{2}\right.$ $\left.-\left(c_{0} / c_{T}^{\infty}\right)^{2}\left(\mathcal{P}_{12}^{\infty}\right)^{2}\right]$.

For the $H H$ spectrum let us just mention that for $\theta$ $=\pi / 2$, Eq. (23) simplifies considerably, since the scalar scattering drops out completely. For frequencies $\omega \tau_{\alpha} \ll 1$, $a_{V H}(z) \rightarrow i a_{V H}^{\prime \prime}$ holds again, and one finds

$$
I_{H H}\left(q, \theta=\frac{\pi}{2}, \omega\right)_{\text {hy }}=\mathcal{T}^{\prime \prime}(\omega=0)-\left(\omega a_{V H}^{\prime \prime}\right)^{2} C_{n n}(q, \omega)^{\prime \prime} .
$$

The hydrodynamic modes are suppressed by a factor of $\omega^{2}$, e.g., the Brillouin line cuts a Lorentzian with half-width $q^{2} \Gamma_{l}$ and amplitude $N\left(a_{V H}^{\prime \prime}\right)^{2} /\left(m \Gamma_{l}\right)$ out of a flat background.

\section{Intensity ratios}

Interesting intensity ratios can be constructed if the scattered intensities with different polarizations of the light before and after the scattering process are considered. The standard depolarization ratio compares the off-resonance $(\omega$ $\gg c q$ ) frequency-dependent background intensities neglecting all hydrodynamic modes:

$$
\frac{I_{V H}(q, \theta, \omega)}{I_{V V}(q, \theta, \omega)}=\frac{\mathcal{T}^{\prime \prime}(\omega)}{\left(\widetilde{Q} s_{00}(\omega) \mid \widetilde{Q} s_{00}\right)^{\prime \prime}+4 \mathcal{T}^{\prime \prime}(\omega) / 3} \leqslant 3 / 4
$$

The expected depolarization ratio $3 / 4$ is recovered if the only scalar scattering mechanisms are density and temperature fluctuations.

\section{COMPARISON WITH PHENOMENOLOGICAL APPROACHES}

It appears worthwhile to discuss earlier phenomenological approaches within our general framework. Again we shall concentrate on the depolarized spectrum, which has been the focus of a long list of theoretical descriptions.

The original approach of Andersen and Pecora [2] and Keyes and Kivelson [3] captures the spectra as predicted by hydrodynamic theory. The two or three variable approaches in Refs. [2,3] go beyond hydrodynamics as they model the background spectrum as a (sum of) Lorentzian(s). Vaucamps et al. extended this approach by adding a further Maxwell relaxation to the shear modulus [8]. More elaborate models, which identify slower and faster relaxing processes in the shear modulus $K_{s}(t)$, were suggested in Refs. [9-11]. Models with more than two variables can be brought into a form suggested by a simple viscoelastic approximation (VA) to our general result, Eq. (17):

$$
I_{V H}^{\mathrm{VA}}(z) \propto \frac{-1}{z+i \Gamma_{\mathcal{T}}}+\frac{1}{\left[z+i \gamma_{V H}\right]^{2}} \frac{-A}{z-q^{2} c_{T}^{2} /\left[z+i / \tau_{s}\right]},
$$

where $A=\left(q a_{V H}^{\infty} \cos \theta / 2\right)^{2} N /\left(m \mathcal{T}^{\infty}\right)$ and Eq. (46) gives a simplified $\alpha$-process only description akin to Maxwell's model. The multivariable phenomenological models, however, rest on specific assumptions about the rotationaltranslational coupling in order to obtain closed equations of motion. Such additional equations, which are not fully constraint by hydrodynamics or symmetry, are not required in our approach. Because of their specific choices, the models in Refs. [9-11] introduced constraints on the coupling parameters in order to describe spectra in supercooled liquids; see Ref. [13] for a detailed discussion of this aspect. Note that the $\alpha$-process only model, Eq. (46), is restricted to low frequencies as can be seen from the vanishing damping of the shear waves in glass, see Eq. (41).

The introduction of retardation effects via memory functions in the work of Wang [10] and Dreyfus et al. $[12,13]$ removed constraints on the coupling parameters, and, within the latter phenomenological approach, an expression formally equivalent to Eq. (17) was given; for linear molecules, corresponding microscopic expressions have been suggested [24].

\section{CONCLUSIONS}

In this paper we discussed the light-scattering spectra for a one-component molecular liquid incorporating slow structural relaxation and thus extending the description to supercooled liquids and glasses. In contrast to earlier phenomenological approaches, no assumptions are made on the nature of the scattering mechanisms nor on the origin of the structural relaxation. Molecules of arbitrary shape and polarizabilities are considered.

From our exclusive use of symmetry arguments it follows that any phenomenological approach complying with generalized hydrodynamics, regardless of the physical mechanisms assumed or nonhydrodynamic variables included, must obey the formulas of Secs. II and III. For an analysis of experimental data, our paper provides a framework clarifying the number of required frequency-dependent kernels, their interpretation, and their most general functional forms.

Our results are akin to the theory of neutron scattering where it is shown that, in general, density fluctuation functions are measured without the need for special models of their dynamics $[15,16]$. For example, Eqs. (17) and (37) indicate how the shear viscosity can be measured by light scattering, without specifying what microscopic dynamical mechanisms contribute to the decay of transverse currents.

We give explicit microscopic formulas for the background spectrum and the elasto-optical constants that can serve as a starting point for approximations once a choice for the scattering mechanism is made. For isotropic particles considering dipole-induced-dipole scattering this has been performed in Ref. [19]. Alternatively, computer simulations could be employed. Our central assumption is to consider systems characterized by short-ranged equilibrium correlations. Thus we can neglect the wave-vector dependence in matrix elements describing the structural relaxation leading to Green-Kubo-like formulas for the memory functions as familiar from simple hydrodynamics.

Whereas previous approaches were mainly concerned with the nature of the depolarized spectrum and correspondingly therefore attribute the scalar fluctuations to a combina- 
tion of density and temperature fluctuations, we carefully make the distinction between dielectric fluctuations coupled to hydrodynamic modes and the ones orthogonal thereto. Consequently one obtains a theory that combines conventional Brillouin and Raman scattering. For example, molecular vibrations or rotational motion give rise to scalar as well as tensor scattering, which appears as background to the hydrodynamic resonances and are included in our framework.

An important aspect of our results is that no assumptions on, e.g., translational-rotational coupling or about the concrete description of the structural relaxation were necessary. The general aspects of the light-scattering spectra were worked out and correlation functions were defined, which can, in principle, be measured experimentally, and which contain the general information about the dynamics of the sample under study.

\section{ACKNOWLEDGMENTS}

Valuable discussions with Professor W. Götze and Professor H. Z. Cummins, and their helpful comments on the manuscript, are gratefully acknowledged. This work was supported by the Deutsche Forschungsgemeinschaft under Grant Nos. Fr 417/2 and Fu 309/3.

\section{APPENDIX A: SCATTERING GEOMETRIES}

The scattering plane, i.e., the plane that contains both the wave vectors $\mathbf{k}_{i}, \mathbf{k}_{f}$ of the incident and scattered wave, is chosen as the $x z$ plane. The direction of the momentum transfer $\mathbf{q}=\mathbf{k}_{i}-\mathbf{k}_{f}$ is taken to be $(0,0,-q)$. Since we consider only small frequency shifts $\left|\mathbf{k}_{i}\right| \simeq\left|\mathbf{k}_{f}\right|$ the scattering angle $\theta=\angle\left(\mathbf{k}_{f}, \mathbf{k}_{i}\right)$ is related to the momentum transfer via $q=2 k_{i} \sin (\theta / 2)$. The dielectric fluctuations corresponding to the conventional scattering geometries, namely, polarization perpendicular $(V)$ to and in the scattering plane $(H)$, are then given by [1]

$$
\begin{gathered}
\delta \epsilon_{V V}(\mathbf{q})=\delta \epsilon_{y y}(\mathbf{q}) \\
\delta \epsilon_{V H}(\mathbf{q})=\delta \epsilon_{x y}(\mathbf{q}) \sin \frac{\theta}{2}-\delta \epsilon_{y z}(\mathbf{q}) \cos \frac{\theta}{2} \\
\delta \epsilon_{H V}(\mathbf{q})=\delta \epsilon_{x y}(\mathbf{q}) \sin \frac{\theta}{2}+\delta \epsilon_{y z}(\mathbf{q}) \cos \frac{\theta}{2} \\
\delta \epsilon_{H H}(\mathbf{q})=\delta \epsilon_{x x}(\mathbf{q}) \sin ^{2} \frac{\theta}{2}-\delta \epsilon_{z z}(\mathbf{q}) \cos ^{2} \frac{\theta}{2}
\end{gathered}
$$

The scattering intensities are expressed via the spectra of the dielectric fluctuations

$$
\begin{aligned}
I_{i o}(q, \theta, \omega) & =\left(\epsilon_{i o}(\mathbf{q}, \omega) \mid \epsilon_{i o}(\mathbf{q})\right)^{\prime \prime} \\
& =\int_{0}^{\infty} d t \cos (\omega t)\left(\epsilon_{i o}(\mathbf{q}, t) \mid \epsilon_{i o}(\mathbf{q})\right) .
\end{aligned}
$$

The scattering intensities obey the sum rule

$$
\frac{1}{\pi} \int_{-\infty}^{\infty} d \omega I_{i o}(q, \theta, \omega)=I_{i o}(q, \theta)
$$

where the total intensities $I_{i o}(q, \theta)$ are determined by the thermal fluctuation of the dielectric tensors

$$
I_{i o}(q, \theta)=\left(\epsilon_{i o}(\mathbf{q}, t=0) \mid \epsilon_{i o}(\mathbf{q})\right)=\frac{\left\langle\left|\delta \epsilon_{i o}(\mathbf{q})\right|^{2}\right\rangle}{k_{B} T} .
$$

\section{APPENDIX B: GENERALIZED HYDRODYNAMICS}

The correlation functions in the generalized hydrodynamic limit can be expressed as [14]

$$
\begin{aligned}
C_{n n}(q, z)= & -N n \kappa_{T} /\left\{z-\left(c_{0} q\right)^{2} /\left\{z+q^{2} K_{l}(z) /(m n)\right.\right. \\
& \left.\left.-q^{2} m T \beta(z)^{2} /\left[z c_{V}(z)+q^{2} \lambda(z)\right]\right\}\right\}, \\
C_{n \Theta}(q, z)=- & N T q^{2} \beta(z) /\left\{\left[z^{2}-\left(c_{0} q\right)^{2}+z q^{2} K_{l}(z) /(m n)\right]\right. \\
\times & {\left.\left[z c_{V}(z)+q^{2} \lambda(z)\right]-q^{2} m T z \beta(z)^{2}\right\}, } \\
C_{\Theta \Theta}(q, z)= & -N T /\left\{z c_{V}(z)+q^{2} \lambda(z)-q^{2} m T z \beta(z)^{2} /\right. \\
& {\left.\left[z^{2}-\left(c_{0} q\right)^{2}+z q^{2} K_{l}(z) /(m n)\right]\right\} . }
\end{aligned}
$$

Here $c_{0}=\left(m n \kappa_{T}\right)^{-1 / 2}$ denotes the isothermal sound velocity, $K_{l}(z)=\left(\tau^{L}\left|R^{\prime}(z)\right| \tau^{L}\right) n / N$ the longitudinal stress relaxation kernel, and $\lambda(z)=\left(j_{e}^{L}\left|R^{\prime}(z)\right| j_{e}^{L}\right) /(N T)$ the thermal conductivity. The frequency-dependent expansion coefficient and specific heat are given by

$$
\begin{gathered}
\beta(z)=\beta+z\left(p\left|R^{\prime}(z)\right| e^{P}\right) /(N m T), \\
c_{V}(z)=c_{V}+z\left(e^{P}\left|R^{\prime}(z)\right| e^{P}\right) /(N T),
\end{gathered}
$$

where $\beta=\left(p \mid Q_{n} e\right) /(N m T), c_{V}=\left(e \mid Q_{n} e\right) /(N T)$ retain their standard values.

There appear three hydrodynamic modes in the liquid state. First, the sound doublet $z= \pm c q-i q^{2} \Gamma_{l} / 2+\mathcal{O}\left(q^{3}\right)$ with the adiabatic sound velocity, $c^{2}=c_{0}^{2}+m T \beta^{2} / c_{V}$ and

$$
\Gamma_{l}=\frac{K_{l}^{\prime \prime}}{n m}+\frac{m T \beta^{2}}{c_{V}}\left[\frac{\lambda^{\prime \prime}}{c^{2} c_{V}}+\frac{c_{V}^{\prime \prime}}{c_{V}}-2 \frac{\beta^{\prime \prime}}{\beta}\right]
$$

where we have written $i K_{l}^{\prime \prime}=K_{l}(z \rightarrow 0), i \lambda^{\prime \prime}=\lambda(z \rightarrow 0), c_{V}(z$ $\rightarrow 0)=c_{V}+i z c_{V}^{\prime \prime}, \quad \beta(z \rightarrow 0)=\beta+i z \beta^{\prime \prime}$. In the generalized hydrodynamic approach the sound damping consists of four parts: viscous friction, losses at conversion from mechanical into thermal energy, losses from storing and extracting thermal energy, and thermal diffusion. Although the expression looks unfamiliar, the associated Green-Kubo formula, Eq. (36), shows that the longitudinal viscosity appears, leading to the well-known result $[7,15]$. Second, there is the heat mode $z=-i q^{2} D_{T}$, where the thermal diffusion constant reads 


$$
D_{T}=\frac{c_{0}^{2}}{c^{2}} \frac{\lambda^{\prime \prime}}{c_{V}}=\frac{\lambda^{\prime \prime}}{c_{P}}
$$

For the second equality in Eq. (B7) we made use of standard thermodynamic transformation formulas. The merit of the generalized hydrodynamic approach lies in the separation of the long-wavelength and low-frequency properties [14]. The only wave-vector dependence arises from the conservation laws. The structural relaxation is captured in generalized transport and thermodynamic derivatives, which exhibit a significant frequency dependence in the regime of interest.

Let us finally highlight the advantage of measuring the fluctuating temperature from the fast kinetic-energy fluctuations by quoting the resulting fluctuations of the heat energy $\tilde{q}(\mathbf{q})=Q_{n} e(\mathbf{q})-T m \beta n(\mathbf{q})[15,18]$. Using the identity Eq. (24) from Ref. [14] one immediately finds:

$$
\begin{aligned}
C_{\tilde{q} \tilde{q}}(q, z)= & \frac{-N T\left[c_{P}-c_{V}(z)-\left(c_{P}-c_{V}\right) \beta^{2}(z) / \beta^{2}\right]}{z} \\
& +c_{V}^{2}(z) C_{\Theta \Theta}(q, z)-2 m T \beta(z) c_{V}(z) C_{n \Theta}(q, z) \\
& +[m T \beta(z)]^{2} C_{n n}(q, z)
\end{aligned}
$$

which in the fluid only exhibits the heat mode characterized by Eq. (B7). In the nonergodic glass, an amplitude $\tilde{q}^{\infty}=1$ $-c_{V}^{\infty} \gamma^{\infty} / c_{V} \gamma$ of the heat fluctuations arrests together with the structural motion, and only the remaining part, $1-\tilde{q}^{\infty}$, relaxes via heat diffusion characterized by $D_{T}^{\infty}$.

\section{APPENDIX C: SPECIAL SCATTERING MECHANISMS}

In order to show that all contributions to the lightscattering spectra discussed in the text will be present in general, and also in order to exemplify how our general formulas can be used, specific scattering mechanisms are listed:

(1) If single scattering processes from biaxial (likely chiral) molecules are considered, the background spectrum arises from the two irreducible spherical tensors:

$$
\begin{gathered}
s_{00}=\frac{1}{3}\left(\alpha_{x x}+\alpha_{y y}+\alpha_{z z}\right) n(\mathbf{q}), \quad q \rightarrow 0, \\
t_{2 \nu}=\frac{1}{\sqrt{8}}\left(\alpha_{x x}-\alpha_{y y}\right) \sum_{i}\left[D_{\nu, 2}^{(2)}\left(\Omega_{i}\right)+D_{\nu,-2}^{(2)}\left(\Omega_{i}\right)\right] \\
+\frac{1}{\sqrt{12}}\left(2 \alpha_{z z}-\alpha_{x x}-\alpha_{y y}\right) \sum_{i} D_{\nu, 0}^{(2)}\left(\Omega_{i}\right),
\end{gathered}
$$

where the scalar dynamics follows the density fluctuations and the Wigner functions $D_{\nu \mu}^{(j)}(\Omega)$ capture the dynamics of the molecular orientation in terms of Euler angles $\Omega$ $=(\varphi, \vartheta, \chi)[25]$.

(2) In the special case of linear molecules, $\alpha_{x x}=\alpha_{y y}$, the tensor fluctuations simplify

$$
t_{2 \nu}=\sqrt{\frac{4 \pi}{15}}\left(\alpha_{z z}-\alpha_{x x}\right) \sum_{i} Y_{2 \nu}\left(\theta_{i}, \varphi_{i}\right)
$$

and the standard spherical harmonics appear [1].

(3) In the case of optically isotropic particles, depolarized scattering can arise from first order dipole-induced-dipole (DID) scattering [26]:

$$
t_{2 \nu}=\frac{\alpha^{2}}{\sqrt{2}} \int \frac{d^{3} k}{(2 \pi)^{3}} n(-\mathbf{k}) T_{2 \nu}(\hat{\mathbf{k}}) n(\mathbf{k}),
$$

where $T_{2 \nu}(\hat{\mathbf{k}})=-4 \pi \sqrt{8 \pi / 15} Y_{2 \nu}(\hat{\mathbf{k}})$ is the static dipole tensor.

(4) If second-order DID scattering of isotropic particles is considered, then the scalar scattering consists of a contribution coupling to the density fluctuations, Eq. (C1), and of another nonhydrodynamic contribution:

$$
\begin{aligned}
& s_{00}= \frac{\alpha^{3}}{3} \sum_{\mu} \int \frac{d^{3} k d^{3} p}{(2 \pi)^{6}}(-1)^{\mu} T_{2, \mu}(\hat{\mathbf{k}}) \\
& \times T_{2,-\mu}(\hat{\mathbf{p}}) n(-\mathbf{k}-\mathbf{p}) n(\mathbf{k}) n(\mathbf{p}), \\
& t_{2 \nu}=-\sqrt{\frac{35}{24}} \alpha^{3} \sum_{\mu}(-1)^{\nu}\left(\begin{array}{ccc}
2 & 2 & 2 \\
\mu & \nu-\mu & -\nu
\end{array}\right) \\
& \times \int \frac{d^{3} k d^{3} p}{(2 \pi)^{6}} T_{2, \nu-\mu}(\hat{\mathbf{k}}) T_{2, \mu}(\hat{\mathbf{p}}) n(-\mathbf{k}-\mathbf{p}) n(\mathbf{k}) n(\mathbf{p}) .
\end{aligned}
$$

This implies that in this case the depolarization ratio from Eq. (45) is smaller than the expected value 3/4. Furthermore the scalar fluctuations overlap with the orthogonalized energy, i.e., $\left(s_{00} \mid Q_{n} e\right)$ is nonzero, and the coupling of dielectric fluctuations to temperature are relevant. In particular, one finds that even in the hydrodynamic limit for a liquid, the Landau-Placzek ratio is not fulfilled.

(5) The lower depolarization ratio also already arises from first order DID of linear molecules:

$$
\begin{aligned}
& s_{00}=\frac{2}{3} \alpha\left(\alpha_{z z}-\alpha_{x x}\right) \sum_{\mu}(-1)^{\mu} \\
& \times \int \frac{d^{3} k}{(2 \pi)^{3}} T_{2,-\mu}(\hat{\mathbf{k}}) n(-\mathbf{k}) n_{2 \mu}(\mathbf{k}), \\
& t_{2 \nu}=-\sqrt{\frac{35}{6}} \alpha\left(\alpha_{z z}-\alpha_{x x}\right) \sum_{\mu} \int \frac{d^{3} k}{(2 \pi)^{3}}(-1)^{\nu} \\
& \times\left(\begin{array}{ccc}
2 & 2 & 2 \\
\mu & \nu-\mu & -\nu
\end{array}\right) T_{2, \nu-\mu}(\hat{\mathbf{k}}) n(-\mathbf{k}) n_{2 \mu}(\mathbf{k}),
\end{aligned}
$$


where the fluctuating tensor density reads $n_{2 \mu}(\mathbf{k})$ $=\sqrt{8 \pi / 15} \Sigma_{i} Y_{2 \mu}\left(\theta_{i}, \varphi_{i}\right) \exp \left(-i \mathbf{k} \cdot \mathbf{r}_{i}\right)$.

(6) If the molecules are not considered as rigid there may be Raman active modes due to intramolecular vibrations. If $Q_{a, i}$ is the vibrational coordinate and $\left(\partial \alpha / \partial Q_{a}\right)$ the corresponding change of the polarizability for mode $a$ there is a contribution to the scalar dielectric fluctuations

$$
s_{00}=\sum_{i} \sum_{a} \frac{\partial \alpha}{\partial Q_{a}} Q_{a, i}
$$

Here the sums run over all molecules and Raman modes. A similar expression can be derived for the dielectric tensor fluctuations.
[1] B. J. Berne and R. Pecora, Dynamic Light Scattering (Wiley, New York, 1976).

[2] H. C. Andersen and R. Pecora, J. Chem. Phys. 54, 2584 (1971).

[3] T. Keyes and K. Kivelson, J. Chem. Phys. 54, 1786 (1971).

[4] V. S. Starunov, E. V. Tiganov, and I. L. Fabelinskii, Zh. Éksp. Teor. Pis'ma Red. 5, 317 (1967) [JETP Lett. 5, 260 (1967)].

[5] C. I. A. Stegeman and B. P. Stoicheff, Phys. Rev. Lett. 21, 202 (1968).

[6] R. D. Mountain, J. Res. Natl. Bur. Stand., Sect. A 70, 207 (1966)

[7] E. M. Lifshitz, L. P. Pitaevskii, and L. D. Landau, Electrodynamics of Continua (Elsevier Science Ltd, New York, 1985).

[8] C. Vaucamps, J. P. Chabrat, L. Letamendia, G. Nouchi, and J. Rouch, J. Phys. (France) 37, 1197 (1976).

[9] B. Quentrec, Phys. Rev. A 15, 1304 (1977).

[10] C. H. Wang, Mol. Phys. 41, 541 (1980); 58, 497 (1986).

[11] P. J. Chapell, M. P. Allen, P. I. Hallen, and D. Kivelson, J. Chem. Phys. 74, 5929 (1981).

[12] C. Dreyfus, A. Aouadi, R. M. Pick, T. Berger, A. Patkowski, and W. Steffen, Europhys. Lett. 42, 55 (1998).

[13] C. Dreyfus, A. Aouadi, R. M. Pick, T. Berger, A. Patkowski, and W. Steffen, Eur. Phys. J. B 9, 401 (1999).
[14] W. Götze and A. Latz, J. Phys.: Condens. Matter 1, 4169 (1989).

[15] D. Forster, Hydrodynamic Fluctuations, Broken Symmetry, and Correlation Functions (W.A. Benjamin, Reading, MA, 1975).

[16] J. P. Hansen and I. R. McDonald, Theory of Simple Liquids (Academic, London, 1986).

[17] P. Scheidler, W. Kob, A. Latz and J. Horbach (unpublished).

[18] L. P. Kadanoff and P. C. Martin, Ann. Phys. (N.Y.) 24, 419 (1963).

[19] M. Fuchs and A. Latz, J. Chem. Phys. 95, 7074 (1991).

[20] S. Watson and P. A. Madden, J. Chem. Phys. 99, 6449 (1993).

[21] W. Götze and L. Sjögren, Rep. Prog. Phys. 55, 241 (1992).

[22] N. V. Surovtsev, J. A. H. Wiedersich, N. V. Novikov, E. Rössler, and A. P. Sokolov, Phys. Rev. B 58, 14888 (1998).

[23] N. J. Tao, G. Li, and H. Z. Cummins, Phys. Rev. Lett. 66, 1334 (1991); H. Z. Cummins, G. Li, Y. H. Wang, G. Q. Shen, W. M. Du, J. Hernandez, and N. J. Tao, Z. Phys. B: Condens. Matter 103, 501 (1997).

[24] A. Latz and M. Letz, Eur. Phys. J. B 19, 345 (2001).

[25] C. G. Gray and K. E. Gubbins, Theory of Molecular Fluids (Clarendon, Oxford, 1984), Vol. 1.

[26] W. M. Gelbart, Adv. Chem. Phys. 24, 1 (1974). 